Revision of accepted manuscript BES-07-0379

\title{
Postcopulatory sexual selection favours intrinsically good sperm competitors
}

Jonathan P. Evans ${ }^{1} \cdot$ Alison N. Rutstein ${ }^{2}$

1. Centre for Evolutionary Biology, School of Animal Biology, University of Western Australia, Nedlands, 6009, WA, Australia

2. School of Biological, Earth and Environmental Sciences, University of New South Wales, Kensington, 2052, NSW, Australia

Correspondence: Jonathan P. Evans, School of Animal Biology (M092), University of Western Australia, Nedlands, 6009, WA, Australia; Email:

jonevans@cyllene.uwa.edu.au 


\section{ABSTRACT}

We used guppies to study repeatability in sperm competitiveness and postcopulatory sexual selection on male ornamentation. In a block design involving 25 pairs of males, artificial insemination was used to mate each pair (A and B) to four unrelated females, two that were mated with the combined ejaculates of both males (sperm competition treatment), and two receiving sperm from each of the two males individually (single male treatment). Our analysis revealed significantly repeatable patterns of paternity across females in the sperm competition treatment, suggesting that certain males are intrinsically better sperm competitors than others, irrespective of female identity. Next, we compared mean brood success (number of offspring per brood) between sperm competition and single male treatments. We found no significant effect of treatment on female fecundity, suggesting that the previously reported direct benefits of polyandry in this species may be due to factors such as differential maternal effects or differences in the number of inseminated sperm between treatments. Our artificial insemination assay was designed to control both factors. Finally, we determined whether variation in relative paternity was random with respect to male phenotype. Unlike previous work on Trinidadian populations, we found no significant relationship between male sexual ornamentation and sperm competitiveness in the focal population.

Keywords: sperm competition, repeatability, Poecilia reticulata, cryptic female choice, good sperm, sexy sperm, sexual ornaments 


\section{INTRODUCTION}

A number of studies have reported that postcopulatory sexual selection has the potential to reinforce or counteract male ornament evolution, depending on the direction of the relationship between ornament size and sperm competitive ability. For example, studies on birds, mammals and fishes have reported either positive or negative phenotypic correlations between components of sperm quality or sperm production and traits involved in mate acquisition (e.g. Matthews et al. 1997; e.g. Froman et al. 2002; Pizzari et al. 2004; Malo et al. 2005). Others have gone further by demonstrating significant associations between male phenotypic traits and $\mathrm{P}_{2}$, defined as the proportion of offspring sired by the second of two males to sequentially mate with a female (Boorman and Parker 1976). For example, several insect studies have reported positive associations between male body size and $\mathrm{P}_{2}$ (Lewis and Austad 1990; LaMunyon and Eisner 1993; Wedell and Cook 1998; Bangham et al. 2002). Since large males often have a mating advantage (Andersson 1994), these studies suggest that postcopulatory sexual selection can augment precopulatory sexual selection on male body size (see also McLain 1991). By contrast, other studies have reported that pre- and postcopulatory sexual selection can act antagonistically on male phenotypic traits. In water striders (Gerris lacustris), for example, relatively large males have a mating advantage but postcopulatory selection appears to favour smaller individuals (Danielsson 2001), possibly due to a trade-off between mating frequency and the number of sperm available at each mating (e.g. Preston et al. 2001).

The association between male ornament size and $\mathrm{P}_{2}$ (whether positive or negative) suggests that sperm competitiveness will be consistently biased in favour of males with particular phenotypic traits. A corollary of this argument is that $\mathrm{P}_{2}$ will be repeatable across different females. Surprisingly, however, only a handful of studies 
have examined repeatability in sperm competition success by analysing variation in $\mathrm{P}_{2}$ after sequentially mating pairs of males to two or more females (Lewis and Austad 1990; Wilson et al. 1997; Radwan 1998; Arnqvist and Danielsson 1999; House and Simmons 2005). These studies, performed on insects, have revealed significant repeatabilities in relative paternity success, suggesting that individual males differ consistently in their ability to compete for fertilizations. Even fewer studies have addressed the possibility that intrinsic differences in male sperm competitiveness reflect differences in the expression of male traits subject to sexual selection (Arnqvist and Danielsson 1999; House and Simmons 2005). This is surprising because such studies can offer important insights into how pre- and postcopulatory sexual selection interact to influence male trait evolution (see reviews by Birkhead and Pizzari 2002; Pizzari et al. 2002; see reviews by Andersson and Simmons 2006).

The guppy (Poecilia reticulata) is an excellent vertebrate model for studying mate choice, polyandry and postcopulatory sexual selection. Females exhibit strong sexual preferences for brightly coloured males, typically preferring males with large bright orange spots (Houde 1997; Brooks and Endler 2001). The area of these orange spots provides information about male condition and may therefore function as an indicator of male genetic quality (van Oosterhout et al. 2003). Female multiple mating (polyandry) is common in laboratory (Evans and Magurran 2000; Pitcher et al. 2003; Becher and Magurran 2004) and natural populations (Kelly et al. 1999) and has been shown to generate postcopulatory sexual selection on male secondary sexual traits. For example, studies on fish descended from Trinidadian populations confirm that both sperm competition (Evans and Magurran 2001; Evans et al. 2003; Pitcher et al. 2003) and cryptic female choice (Pilastro et al. 2004) favour males exhibiting preferred behavioural and ornamental traits (see also Becher and Magurran 2004). 
The current study determines whether relative paternity success is an intrinsic characteristic of particular males, which although documented in several insect studies (cited above) has never (to our knowledge) been shown in vertebrates. We address this question using a block design, where each block comprised two sperm competition trials involving a pair of males mated simultaneously (through artificial insemination) to two unrelated females. Within each block we also incorporated two single male matings, where each of the two males was mated (artificially) to two further females individually. By ensuring that the number of sperm inseminated in the sperm competition and single male treatments were identical, we were able to determine whether female fecundity is influenced by the number of males per se, when mating order and the relative contribution of sperm from both males are controlled (see discussions by Evans and Magurran 2001; Pilastro et al. 2004). Finally, we ask whether postcopulatory sexual selection favours the same traits that are selected during precopulatory sexual selection, which in the focal population include the proportion of orange and iridescent spots and tail size (Brooks and Endler 2001).

\section{METHODS}

\section{Origin and maintenance of fish}

Guppies were laboratory reared descendents of fish caught in 2002 from the Alligator Creek in Bowling Green National Park, Queensland, Australia. Six-month old virgin females were reared for the experiment in several single-sex cultures as soon as they could be sexed (approx. 40 d). Males came from mixed sex stock aquaria. All fish were fed Artemia nauplii throughout the experiment, and were maintained at a constant $26^{\circ} \mathrm{C}$ with a $12: 12 \mathrm{~h}$ light dark cycle. 


\section{Artificial Insemination}

The experiment involved 25 blocks, each containing two focal (unrelated) rival males (denoted A and B) and four unrelated females (1-4). Each block comprised two (repeated) sperm competition trials (male A and male B mated simultaneously through artificial insemination to females 1 and 2) and two single mating treatments (males A and B mated individually to females 3 and 4, respectively). In each of these blocks, sperm were manually stripped from the two males following Matthews et al. (1997). Briefly, each anaesthetised male was placed on a glass slide under low-power magnification with its gonopodium swung forward beyond $90^{\circ}$. Gentle pressure was applied to the abdomen to release spermatozeugmata (sperm bundles). Each sperm bundle contains approximately 30,000 sperm cells (Billard 1969) and previous work on Trinidadian guppies has confirmed that variation in the number of sperm per bundle is no greater among than within males (Evans et al. 2003). It is therefore straightforward to control the relative number of sperm per inseminate by counting the number of sperm bundles.

In each block, three sperm samples (comprising known numbers of sperm bundles - see below) were taken from each male’s stripped ejaculate for the monandrous and polyandrous crosses. Each of these samples was immediately suspended in $8 \mu \mathrm{l}$ of physiological solution $(0.9 \% \mathrm{NaCl})$. Importantly, the three samples were retrieved from each male’s stripped ejaculate independently and were maintained separately until used. We therefore ensured that the two samples of each male's ejaculate used to estimate repeatability could be considered as independent 'ejaculates'. (Note that our UNSW Animal Ethics Approval AEC/015 did not enable us to repeatedly strip each male on separate occasions.) Two of these stripped ejaculates (each containing 10 
sperm bundles) from each male were subsequently used for the repeated sperm competition trials (AB1 and AB2) while the remaining ejaculate (20 sperm bundles) was used for the single-male treatment (i.e. A3 and B4 for males A and B, respectively). In single-male inseminations, the 20 sperm bundles were suspended in $8 \mu \mathrm{l}$ of saline solution and artificially inseminated into each of the two virgin females, while in the sperm competition trials 10 bundles from each male were combined and suspended in $8 \mu \mathrm{l}$ saline and inseminated simultaneously. Hence, across the four inseminations in each block the volume of inseminate was fixed at $8 \mu \mathrm{l}$ and the number of sperm per inseminate was fixed at 20 sperm bundles (containing approx. $5.4 \times 10^{5}$ sperm cells), which is within range for the number of sperm contained in natural ejaculates in guppies (Pilastro and Bisazza 1999; Pilastro et al. 2002). In all cases, extreme care was taken not to break up the sperm bundles in each sample, as this greatly reduces the fertilization capacity of inseminated sperm (J. P. Evans, unpublished observations). Ejaculates from each male within a pair were collected within 30 seconds of each other and used within 5 minutes of collection. Immediately after sperm extraction, a digital photograph was taken of each male for the ornamentation measures (see below). Following this, a small clipping (ca. 25 $\mu$ g) was removed from each male’s caudal fin for the paternity analysis. Males were then revived in conditioned fresh water and released into post experimental tanks where they took no further part in the experiment.

For each insemination, a virgin female (ca. 6 months old, mean standard length = $21.48 \mathrm{~mm} \pm 0.2 \mathrm{~mm}$ s.e.m.) was anaesthetised and placed in a polystyrene cradle with her genital pore exposed. A machine-pulled (Clark Electromedical Instruments) plastic micropipette was used to inject the stripped ejaculates from the two males simultaneously (polyandrous treatments) or individually (single male treatments) into 
the female's gonoduct (penetration depth approximately $2 \mathrm{~mm}$ ). Immediately after artificial insemination, females were revived in conditioned freshwater and isolated in 5L tanks (containing an airstone and artificial weed) until they produced their first brood. After giving birth, a small clipping was taken from each female's caudal fin to facilitate the paternity analysis (see below). Offspring were too small to survive fin clipping and were therefore euthanased in a lethal dose of the anaesthetic MS222 for tissue extraction immediately after the offspring performance measures (see below).

\section{Male ornamentation}

UTHSCSA Image Tool (University of Texas Health Science Center, San Antonio, TX, available at http://ddsdx.uthscsa.edu/dig/itdesc.html) was used to estimate the body area of each male (including caudal fin but excluding dorsal fin), body size (snout to tip of caudal peduncle = standard length), tail area and the surface area of the various colour spots. These colour spots were measured on the left side of each male's body from digital photographs, and included the relative area of carotenoid and pteridine pigments (orange, yellow and red spots, summed as 'orange'), structural colours such as iridescent blue, green, purple, silver and white (summed as (iridescence') and melanin spots and fuzzy black lines (summed as 'black'). Hence, in our analysis of postcopulatory sexual selection on male ornamentation (see Statistical analyses, below), we included three separate colour measures: orange, iridescence and black, as well as standard length and tail area. To control for differences in body size among males, which was significantly positively correlated with the area of black $\left(r_{36}=0.46, p=0.004\right)$ and iridescent $\left(r_{36}=0.40, p=0.015\right)$ colour spots, but not orange $\left(r_{36}=0.06, p=0.71\right.$ ), we corrected for body size between rival males in our subsequent analysis (see below) by using the residuals from separate regressions of black and 
iridescence on male standard length. This provided size-free estimates of male ornamentation for these traits.

\section{Paternity assignment}

Silver staining was used to visualise PCR products for paternity assignment. Five microsatellite markers were used to assign paternity, including TTA (Genbank accession number AF164205; JS Taylor unpubl. cited in Lindholm et al. 2005), KonD15 and KonD21 (respectively AF368429 \& AF368430; Seckinger et al. 2002), Pret46 (AB100334; Watanabe et al. 2003) and Pr39 (AF467903; Becher et al. 2002). Paternity was assigned to offspring according to allele sharing between the two putative sires, mother and offspring. The five loci were moderately variable, ranging from 3 to 9 alleles in the sample, allowing the unambiguous assignment of 216 out of 273 offspring. Offspring that could not be assigned to either male were excluded from the subsequent analyses (see below).

\section{Statistical analyses}

Twelve of the 25 blocks generated repeated paternity values for a given pair of rival sperm competitors (i.e. where both females produced a brood). Relative paternity is given as the proportion of offspring sired by the male arbitrarily assigned as B in each pair, hereafter referred to as $\mathrm{P}_{\mathrm{B}}$ (Evans et al. 2003). Repeatability estimates were derived from these families using the variance components from a one-way analysis of variance of $\mathrm{P}_{\mathrm{B}}$ among blocks. Variance components from this analysis were then used to calculate the intra-class correlation coefficient $\left(r_{\mathrm{i}}\right)$ as a measure of repeatability in $\mathrm{P}_{\mathrm{B}}$ (following angular transformation) (Lessells and Boag 1987). 
We compared brood size (number of offspring per brood) between mating treatments using a General Linear Mixed Model (GLMM), with treatment entered as the fixed factor and family nested within block as a random effect. In this analysis, block refers to the group of (up to) four broods involving the two focal males (A \& B), while family refers to offspring that belong to the same brood. Not all artificial inseminations were successful (see Results), resulting in an unbalanced design. Estimates of the fixed effects were therefore calculated using restricted maximum likelihood estimation (REML) procedures in SPSS (v. 13).

A generalized linear model with binomial errors and a logit link function was used to determine whether male phenotype (colour, standard length and tail area) accounted for significant deviance in $\mathrm{P}_{\mathrm{B}}$. In this analysis, the residual deviance was greater than binomial expectation; the Williams procedure (Williams 1982) was therefore used to account for overdispersion (using logistic modelling procedures and the EXTRABIN function in Genstat 9). Each experimental block comprised two sperm competition treatments (the same two males mated to two females). To ensure that all replicates were statistically independent, we used one family from each block; hence where a block contained two $P_{B}$ scores for the same two males ( $n=12$, see below), one family was selected at random for the analysis (total families analysed $n=18$ ). In each replicate, male B's paternity share (total number of offspring sired by male B) was entered as the response variate, while the total number of offspring in each family was entered as the binomial total. The regression was weighted by family size (total number of offspring in each replicate) and included five fitted terms representing differences in trait values between rival males (male B trait minus male A trait) (see also Evans and Magurran 2001; Evans et al. 2003). These traits included male ornamentation (orange, black and iridescence), standard length and tail size. All 
predictor variables (including residuals extracted from linear regressions of black and iridescence on body size) were normally distributed.

\section{RESULTS}

Twelve of the $n=25$ blocks generated $\mathrm{P}_{\mathrm{B}}$ estimates for both females (mean \pm s.e.m. number of offspring produced $=8.84 \pm 0.76$; range $3-18$ ). One-way analysis of variance of (angular transformed) $\mathrm{P}_{\mathrm{B}}$ values revealed significant differences in relative paternity scores among blocks $\left(F_{11,12}=3.77, p=0.016\right)$; the repeatability analysis based on the variance components from this analysis confirmed that specific male pairs exhibited significantly repeatable $\mathrm{P}_{\mathrm{B}}$ scores across the two females (intra-class correlation coefficient $r_{\mathrm{i}}=0.58 \pm 0.20$ s.e.; see also Fig. 1 ).

The broods produced in this experiment were distributed approximately evenly between double male ( $n=35$; mean number of offspring \pm s.e.m. $=8.26 \pm 0.64$ ) and single male groups $(n=31$; mean offspring \pm s.e.m. $=8.52 \pm 0.74)$. Brood size did not significantly differ between treatments (GLMM: $F_{1,64}=0.35, P=0.85$ ).

Finally, unlike previous work on Trinidadian populations (Evans et al. 2003), our analysis of relative male ornamentation revealed no significant effect of male phenotype on the deviance in $\mathrm{P}_{\mathrm{B}}$ (Table 1).

\section{DISCUSSION}

The repeatability estimate for relative paternity revealed here $\left(r_{\mathrm{i}}=0.58\right)$ is among the highest reported for pairs of males mated to two or more unrelated females (Wilson et al. 1997; Radwan 1998; House and Simmons 2005) (see also Lewis and Austad 1990; see also Arnqvist and Danielsson 1999), suggesting that certain males are intrinsically more competitive than others, irrespective of the environment (female) in which their 
sperm compete. However, it is important to note that high repeatability in sperm precedence may also be due to environmental effects, including female-mediated biases in the use of sperm or maternal effects that occur after insemination. While, our design cannot exclude these effects, differential sperm use by females seems unlikely because the experimental design (employing artificial insemination) effectively decoupled pre- and postcopulatory sexual selection, thus denying females the opportunity of assessing each male at mating (c.f. Pilastro et al. 2004). Nevertheless, future quantitative genetic experiments are required to elucidate the genetic basis of traits that regulate sperm competitiveness (see Evans and Simmons 2007).

We found that in two blocks offspring were sired exclusively by one of the two males across both females (Fig. 1). Such 'all-or-nothing' patterns of paternity have been reported previously in guppies following artificial insemination (Evans et al. 2003) but are particularly pronounced following natural copulations with two males (Evans and Magurran 2001; see also Pitcher et al. 2003). During natural (solicited) copulations, males with relatively high levels of orange pigmentation are known to transfer higher numbers of sperm (Pilastro et al. 2002), an effect that is at least partly influenced by females (Pilastro et al. 2004). The recent findings that insemination success is positively associated with copulation duration, and that copulation duration is positively correlated with the extent of orange pigmentation in the male's colour spots (Pilastro et al. 2007), suggests that females have the potential to exert 'cryptic' female choice by adjusting copulation duration to favour preferred males. Such cryptic female preferences may therefore explain the extreme bimodal distribution of paternity following sequential natural copulations in this species (Evans and Magurran 2001). In the current study, however, the use of artificial insemination precluded such female-mediated effects, whilst concomitantly standardizing the 
relative contribution of sperm from rival males. Under these conditions, exclusive paternity by a given male across both females is likely to be due either to differences in the relative competitiveness, or the viability, of sperm from rival males. When the two families (9 \& 12 in Fig. 1) that exhibited all-or-nothing $\mathrm{P}_{\mathrm{B}}$ scores across both females were excluded from our repeatability analysis, we found that relative paternity was moderately repeatable $\left(r_{\mathrm{i}}=0.35\right)$ but within the range of repeatabilities reported for several insects (cowpea weevil $=0.55$ : Wilson et al. 1997; bulb mite = 0.22: Radwan 1998; dung beetle $=0.49$ : House and Simmons 2005).

Surprisingly, our study revealed no significant association between male sexual ornamentation and paternity success. This finding contrasts with previous work on fish descended from the Tacarigua River in Trinidad where a significant positive relationship was found between relative orange pigmentation and sperm competitiveness (Evans et al. 2003). The different origins of the two populations may account for the different results (different populations are known to exhibit different phenotypic relationships between sperm traits and male phenotype; Pitcher and Evans 2001). On the other hand, subtle differences in environmental conditions between studies may have masked phenotypic associations between relative paternity success and male secondary sexual traits. For example, it has been hypothesised that both carotenoid-based ornaments and ejaculate traits are prone to free-radical induced deterioration, which is mitigated by antioxidant levels in the diet (Blount et al. 2001). Hence, the relationship between carotenoid based traits and sperm performance (and therefore presumably competitiveness) may only be apparent under dietary stress. Current work is specifically addressing this question by determining whether dietary stress mediates the relationship between carotenoid-based ornamental traits and sperm quality. 
We also failed to detect a significant effect of mating treatment on female fecundity (brood size). This finding contrasts with previous work on Trinidadian guppies which compared female fecundity between singly- and multiply mated treatments (Evans and Magurran 2000). Evans and Magurran (2000) reported that multiply-mated females produced larger broods comprising offspring with enhanced antipredator capabilities. However, in Evans and Magurran's (2000) study, females mated freely with males and therefore differential maternal effects may have influenced brood traits (e.g. Gil et al. 1999; Cunningham and Russell 2000; Kolm 2001; Gil et al. 2004). Our use of artificial insemination limited the opportunity for such effects in the current study (see also Parker 2003). Furthermore, the influence of mating treatment on female fecundity (brood size) reported by Evans and Magurran (2000) may have been due to females accepting more sperm from preferred males, a phenomenon known to occur in guppies (Pilastro et al. 2004). Again, our artificial insemination protocol prevented such effects in the current investigation. We are currently testing the differential allocation hypothesis in guppies to determine whether the previously documented benefits of polyandry in guppies are due to either (nongenetic) maternal effects or intrinsic (genetic) sire effects.

\section{Acknowledgements}

We thank Megan Head for help with colour scoring, Jennifer Kelley and Lee Ann Rollins for assistance with fish husbandry, Robert Black for statistical advice and Leigh Simmons, Jennifer Kelley and two anonymous referees for helpful comments on the manuscript. Thanks also to Rob Brooks for discussion, logistic support and access to facilities while at the University of New South Wales. JPE gratefully acknowledges financial support from the Australian Research Council. 


\section{REFERENCES}

Andersson M (1994) Sexual Selection. Princeton University Press, Princeton, NJ

Andersson M, Simmons LW (2006) Sexual selection and mate choice. Trends in Ecology and Evolution 21:296-302

Arnqvist G, Danielsson I (1999) Copulatory behavior, genital morphology, and male fertilization success in water striders. Evolution 53:147-156

Bangham J, Chapman T, Partridge L (2002) Effects of body size, accessory gland and testis size on pre- and postcopulatory success in Drosophila melanogaster. Animal Behaviour 64:915-921

Becher SA, Magurran AE (2004) Multiple mating and reproductive skew in Trinidadian guppies. Proceedings of the Royal Society London B 271:10091014

Becher SA, Russell ST, Magurran AE (2002) Isolation and characterization of polymorphic microsatellites in the Trinidadian guppy (Poecilia reticulata). Molecular Ecology Notes 2:456-458

Billard R (1969) La sperm spermatogenese de Poecilia reticulata. Ann. Biol. Anim. Bioch. Biophys. 9:251-271

Birkhead TR, Pizzari T (2002) Postcopulatory sexual selection. Nature Reviews Genetics 3:262-273

Blount JD, Møller AP, Houston DC (2001) Antioxidants, showy males and sperm quality. Ecology Letters 4:393-396

Boorman E, Parker GA (1976) Sperm (ejaculate) competition in Drosophila melanogaster and the reproductive value of females to males in relation to female age and mating status. Ecological Entomology 1:145-155

Brooks R, Endler JA (2001) Female guppies agree to differ: phenotypic and genetic variation in mate-choice behavior and the consequences for sexual selection. Evolution 55:1644-1655

Cunningham EJA, Russell AF (2000) Egg investment is influenced by male attractiveness in the mallard. Nature 404:74-77

Danielsson I (2001) Antagonistic pre- and post-copulatory sexual selection on male body size in a water strider (Gerris lacustris). Proceedings of the Royal Society London B 268:77-81

Evans JP, Magurran AE (2000) Multiple benefits of multiple mating in guppies. Proceedings of the National Academy of Sciences USA 97:10074-10076 
Evans JP, Magurran AE (2001) Patterns of sperm precedence and predictors of paternity in the Trinidadian guppy. Proceedings of the Royal Society London B 268:719-724

Evans JP, Simmons LW (2007) The genetic basis of traits regulating sperm competition and polyandry: can selection favour the evolution of good- and sexy-sperm? Genetica in press

Evans JP, Zane L, Francescato S, Pilastro A (2003) Directional postcopulatory sexual selection revealed by artificial insemination. Nature 421:360-363

Froman DP, Pizzari T, Feltmann AJ, Castillo-Juarez H, Birkhead TR (2002) Sperm mobility: mechanisms of fertilizing efficiency, genetic variation and phenotypic relationship with male status in the domestic fowl, Gallus gallus domesticus. Proceedings of the Royal Society London B 269:607-612

Gil D, Graves J, Hazon N, Wells A (1999) Male attractiveness and differential testosterone investment in zebra finch eggs. Science 286:126-128

Gil D, Leboucher G, Lacroix A, Cue R, Kreutzer M (2004) Female canaries produce eggs with greater amounts of testosterone when exposed to preferred male song. Hormones and Behavior 45:64-70

Houde AE (1997) Sex, Color, and Mate Choice in Guppies. Princeton University Press, Princeton NJ

House CM, Simmons LW (2005) Relative influence of male and female genital morphology on paternity in the dung beetle Onthophagus taurus. Behavioral Ecology 16:889-897

Kelly CD, Godin JGJ, Wright J (1999) Geographic variation in multiple paternity within natural populations of the guppy (Poecilia reticulata). Proceedings of the Royal Society London B 266:2403-2408

Kolm N (2001) Females produce larger eggs for large males in a paternal mouthbrooding fish. Proceedings of the Royal Society London B 268:22292234

LaMunyon CW, Eisner T (1993) Postcopulatory sexual selection in an arctiid moth (Utetheisa ornatrix). Proceedings of the National Academy of Sciences USA 90:4689-4692

Lessells CM, Boag PT (1987) Unrepeatable repeatabilities: a common mistake. Auk 104:116-121

Lewis SM, Austad SN (1990) Sources of intraspecific variation in sperm precedence in red flour beetles. The American Naturalist 135:351-359

Lindholm AK, Breden F, Alexander HJ, Chan WK, Thakurta SG, Brooks R (2005) Invasion success and genetic diversity of introduced populations of guppies Poecilia reticulata in Australia. Molecular Ecology 14:3671-3682 
Malo AF, Roldan ERS, Garde J, Soler AJ, Gomendio M (2005) Antlers honestly advertise sperm production and quality. Proceedings of the Royal Society London B 272:149-157

Matthews IM, Evans JP, Magurran AE (1997) Male display rate reveals ejaculate characteristics in the Trinidadian guppy Poecilia reticulata. Proceedings of the Royal Society London B 264:695-700

McLain DK (1991) Components of variance in male lifetime copulatory and reproductive success in a seed bug. Behavioral Ecology and Sociobiology 29:121-126

Parker TH (2003) Genetic benefits of mate choice separated from differential maternal investment in red junglefowl (Gallus gallus). Evolution 57:21572165

Pilastro A, Bisazza A (1999) Insemination efficiency of two alternative male mating tactics in the guppy (Poecilia reticulata). Proceedings of the Royal Society London B 266:1887-1891

Pilastro A, Evans JP, Sartorelli S, Bisazza A (2002) Male phenotype predicts insemination success in guppies. Proceedings of the Royal Society London B 269:1325-1330

Pilastro A, Mandelli M, Gasparini C, Dadda M, Bisazza A (2007) Copulation duration, insemination efficiency and male attractiveness in guppies. Animal Behaviour 74:321-328

Pilastro A, Simonato M, Bisazza A, Evans JP (2004) Cryptic female preferences for colorful males in guppies. Evolution 58:665-669

Pitcher TE, Evans JP (2001) Male phenotype and sperm number in the guppy (Poecilia reticulata). Canadian Journal of Zoology 79:1891-1896

Pitcher TE, Neff BD, Rodd FH, Rowe L (2003) Multiple mating and sequential mate choice in guppies: females trade up. Proceedings of the Royal Society London B 270:1623-1629

Pizzari T, Froman DP, Birkhead TR (2002) Pre- and post-insemination episodes of sexual selection in the fowl, Gallus g. domesticus. Heredity 88:112-116

Pizzari T, Jensen P, Cornwallis CK (2004) A novel test of the phenotype-linked fertility hypothesis reveals independent components of fertility. Proceedings of the Royal Society London B 271:51-58

Preston BT, Stevenson IR, Pemberton JM, Wilson K (2001) Dominant rams lose out by sperm depletion. Nature 409:681-682

Radwan J (1998) Heritability of sperm competition success in the bulb mite, Rhizoglyphus robini. Journal of Evolutionary Biology 11:321-327 
Seckinger J, Brinkmann H, Meyer A (2002) Microsatellites in the genus Xiphophorus, developed in Xiphophorus montezumae. Molecular Ecology Notes 2:4-6

van Oosterhout C, Trigg RE, Carvalho GR, Magurran AE, Hauser L, Shaw PW (2003) Inbreeding depression and genetic load of sexually selected traits: how the guppy lost its spots. Journal of Evolutionary Biology 16:273-281

Watanabe T, Yoshida M, Nakajima M, Taniguchi N (2003) Isolation and characterization of 43 microsatellite DNA markers for guppy (Poecilia reticulata). Molecular Ecology Notes 3:487-490

Wedell N, Cook PA (1998) Determinants of paternity in a butterfly. Proceedings of the Royal Society London B 265:625-630

Williams DA (1982) Extra-binomial variation in logistic linear models. Applied Statistics 31:144-148

Wilson N, Tubman SC, Eady PE, Robertson GW (1997) Female genotype affects male success in sperm competition. Proceedings of the Royal Society London B 264:1491-1495 


\section{Table 1 Proportion of offspring sired in relation to male phenotype}

Generalized linear model with binomial errors

d.f. deviance $\begin{gathered}\text { mean } \\ \text { deviance }\end{gathered}$ ratio $P$

$\begin{array}{lccccc}\text { Regression } & 5 & 3.44 & 0.69 & 0.69 & 0.63 \\ \text { Residual } & 12 & 15.34 & 1.28 & & \\ \text { Total } & 17 & 18.78 & 1.11 & & \end{array}$

\section{Estimate of parameters}

$\begin{array}{lcccc}\text { Parameter } & \text { estimate } & \text { s.e.m. } & t & P \\ \text { Constant } & -0.414 & 0.532 & -0.78 & 0.437 \\ \text { Difference in orange } & -0.142 & 0.170 & -0.83 & 0.405 \\ \text { Difference in iridescence* } & 0.067 & 0.092 & 0.73 & 0.468 \\ \text { Difference in black* } & 0.189 & 0.209 & 0.91 & 0.365 \\ \text { Difference in standard length } & 0.517 & 0.543 & 0.95 & 0.341 \\ \text { Difference in tail size } & 0.007 & 0.160 & -0.04 & 0.964\end{array}$

*Analysis based on use of unstandardized residuals from regressions of colour scores on body size (see main text). Results remain unchanged when differences between rival males in absolute (rather than relative) colours are analysed. Overdispersion was corrected using the Williams procedure ( $\phi=0.56$, model II, see text for further details). 


\section{Figure legend}

Figure 1 Variance in relative paternity $\left(\mathrm{P}_{\mathrm{B}}\right)$ across $n=12$ pairs of females. The dashed lines around both points are for illustrative purposes and provide an indication of the level of variability in $\mathrm{P}_{\mathrm{B}}$ within and among blocks. In each replicate filled circles denote $\mathrm{P}_{\mathrm{B}}$ scores for broods from female 1, unfilled circles are for broods from female 2. 
Figure 1

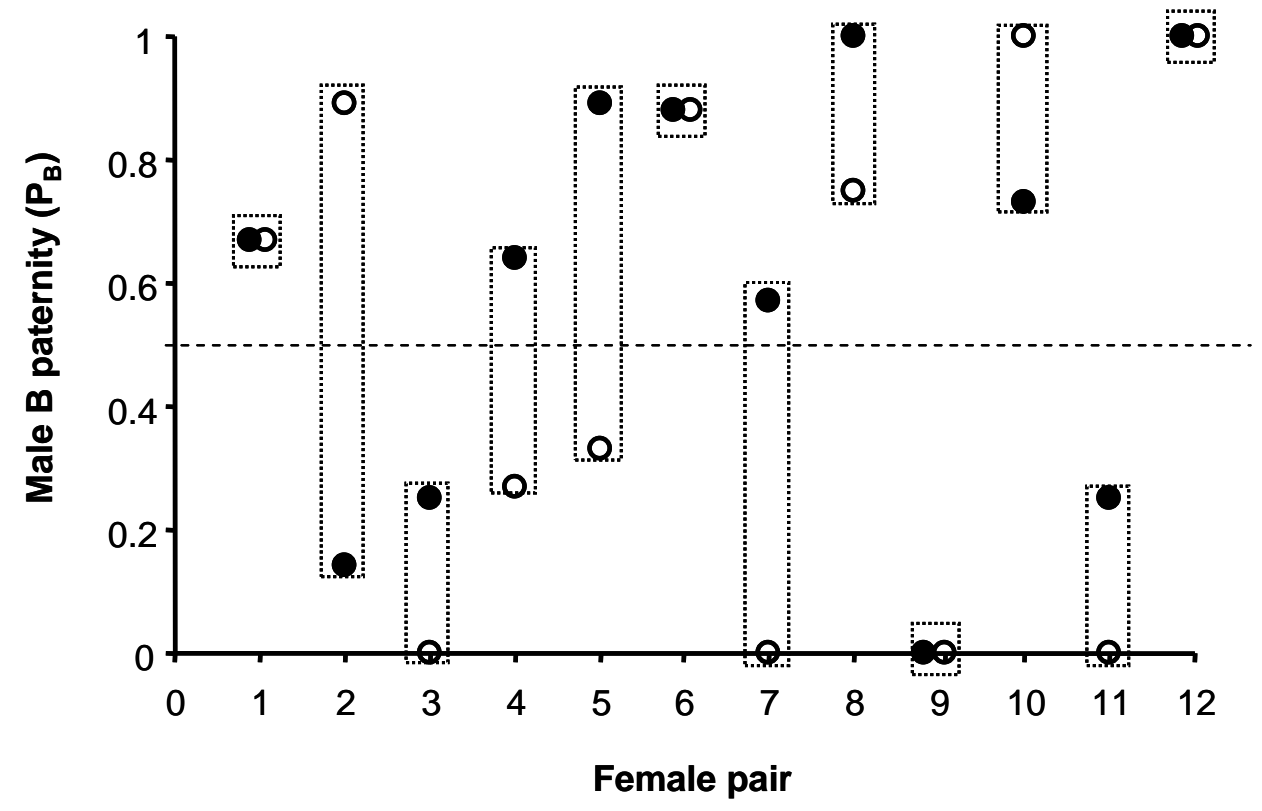

